\title{
Effects of social isolation and crowding upon active-avoidance performance in the rat
}

\author{
M. P. VIVEROS, R. HERNANDEZ, and A. GALLEGO \\ Universidad Complutense, Ciudad Universitaria, Madrid, Spain
}

\begin{abstract}
Male and female Sprague-Dawley rats reared in social isolation and in crowded conditions were tested on their active-avoidance performance. Only males appeared to be affected by the treatments; no housing-condition effect was found on the performance of females. The most severe housing-condition effect was that of social isolation; the isolated males showed impaired acquisition of the avoidance response. We have previously reported diminished adrenocortical flexibility in male isolates, which might account for the present results. A possible failure of the inhibition mechanisms of isolates is also taken into account.
\end{abstract}

Isolation rearing of rats has been found to induce difficulties in inhibiting certain responses in a number of situations, which does not seem to be fully explained by attributing a unitary disinhibition deficit to the isolated animals (Dalrymple-Alford \& Benton, 1984; Joseph \& Gallager, 1980; Morgan \& Einon, 1975). In general, these data suggest a diminished learning ability in isolates. However, some apparent discrepancies exist regarding isolates' performance in the active-avoidance learning paradigm. Thus, several studies indicate that individual housing facilitates such performance (Lovely, Pagano, \& Paolino, 1972), whereas others suggest an impairment (Will \& Kelche, 1979). Unmodified active-avoidance behavior has also been reported in isolates (Eclancher \& Karly, 1980). In the present work, we attempt to clarify this matter. Active-avoidance conditioning is a suitable paradigm for testing the behavioral flexibility necessary for an escape response to an unconditioned stimulus (US) to be replaced by an avoidance response to a conditioned stimulus (CS). According to several previous reports, this behavioral flexibility appears to be damaged in isolationreared animals (Dalrymple-Alford \& Benton, 1984; Joseph \& Gallager, 1980; Morgan \& Einon, 1975). Thus, one might expect to find impaired avoidance learning in isolates.

The other social treatment used in this workcrowding-involved an enhanced social experience, which might act to induce improved learning (Joseph, 1979; Juraska, Henderson, \& Müller, 1984; Rosenzweig, Bennett, Hebert, \& Morimato, 1978; Welch, Brown, Welch, $\&$ Lin, 1974), but the possibility of stress resulting from the crowded living conditions may result in a learning deficit. This raises the question as to which of these two effects would prevail.

Both social treatments, isolation and crowding, appear to influence the function of the pituitary-adrenal axis.

\footnotetext{
We thank Steve Bailey for a critical reading of the manuscript. Address correspondence to M. P. Viveros, Departmento de Biolgía Animal II (Fisiología Animal), Facultad de Biología, Universidad Complutense, Ciudad Universitaria, 28040 Madrid, Spain.
}

Although definitive conclusions may not be deduced about the emotional significance of the adrenal modifications found in crowded animals (Viveros, Hernández, Martínez, \& González, 1988), several studies have found increased emotional reactivity in crowded males (Armario, Castellanos, \& Balasch, 1984; Thiebot, Soubrie, Chermat, Simon, \& Boissier, 1977). In the case of isolates, we have recently suggested, in agreement with previous reports (Plaut \& Grota, 1971), an impaired functional flexibility of the pituitary-adrenal axis (Viveros et al., 1988). This axis plays a major role in a variety of adaptive responses, especially in performances involving intense emotional reactivity such as the one tested in the present work (Wilcock \& Fulker, 1973). Hence, one would expect to find decreased active-avoidance performance in treated animals, particularly in males, as a consequence of a possible increased emotional level (crowded animals) or of a decreased adrenal functional flexibility (isolates).

Finally, since a sexually dimorphic emotional level (Gray, 1979; Gray \& Lalljee, 1974) might influence the learning ability of the animals in this paradigm, the possibility of a differential effect of the social treatments on the emotional reactivity of males and females was also considered.

\section{METHOD}

\section{Animals and Housing Conditions}

A total of 71 male and female Sprague-Dawley rats were used. The animals were kept in a room at approximately $22^{\circ} \mathrm{C}$ with a 12-h reversed light-dark cycle (2000-0800 h light). Food and water were supplied ad libitum. Differential housing was introduced at weaning (22 days of age). The experimental groups were as follows: socially isolated males (SIM, $N=12$ ) and socially isolated females (SIF, $N=11$ ), housed in individual $24 \times 24 \times 15 \mathrm{~cm}$ cages; control males $(C M, N=10)$ and control females $(C F$, $N=10$ ), housed 4-6 animals per cage; and crowded males (CRM, $N=14$ ) and crowded females (CRF, $N=14$ ), housed 14 animals per cage. Socially reared animals (control and crowded rats) were housed in $48 \times 24 \times 15 \mathrm{~cm}$ standard laboratory cages. Individually housed rats could receive several stimuli (other than tactile) from the other animals living in the same room. Thus, the isolation was only considered as being "social." 


\section{Apparatus}

The two-way shuttlebox apparatus was a $40 \times 23 \times 30 \mathrm{~cm}$ rigid, transparent plastic box with a grid floor connected to a DC power supply. The floor was divided into two compartments by an $8-\mathrm{cm}$ bar. Each compartment had a $\mathbf{4 0 - W}$ bulb located on its lateral wall, $25 \mathrm{~cm}$ above the grid. The illumination and electric shock were independent for each compartment. A continuous background noise was provided by an electric fan during the test.

\section{Procedure}

At 75 days of age, the animals were individually subjected to a 10-day initial phase (acquisition), with 5-min daily sessions. Each experimental session consisted of 10 trials with the CS-US sequence being presented at 30-sec intervals. At 140 days, each of the animals was tested for retention, this time for 5 consecutive days. Identical 5 -min sessions were used in this second stage of the test. The increase in body weight of the animals between the two phases was taken into consideration. Thus, $60-\mathrm{V}$ electric shocks (US) were delivered during acquisition, and a higher voltage $(70 \mathrm{~V})$ was used during the retention phase. The $40-\mathrm{W}$ illumination previously described was used as the CS. The CS was presented alone for $5 \mathrm{sec}$. The US was subsequently superimposed for $3 \mathrm{sec}$ (maximum shock duration). If the rat jumped over the bar to the other compartment (free of stimuli) during the first $5 \mathrm{sec}$, the light was extinguished and shock was avoided. If the animal jumped during the last $3 \mathrm{sec}$, both stimuli were extinguished.

The following responses were recorded: (1) avoidance response (AVO), in which the animal jumped into the "harmless" compartment during the application of the CS (before receiving the electric shock), and (2) escape response (ESC), in which the animal jumped over the bar only after the electric shock was presented. The number of errors ( $E$; when the animal remained in the same compartment without responding to either stimulus) and the defecation score (Def; number of boluses) during each session were also recorded.

\section{Statistical Analysis}

Data analysis was carried out using a three-way analysis of variance (ANOVA), which allowed for discrimination and evaluation of the contributions of each main factor (i.e., housing condition, sex, and repeated measures on trials) to the total variance, as well as the double and triple interactions among the factors. The variables studied were AVO and ESC responses, and Def scores. The results for the first two variables were highly correlated, because from Day 3 in the acquisition phase, they add up to 10 in all groups except SIM; therefore, the results in the corresponding analysis were interpreted as a whole.

The data obtained for $\mathrm{E}$ revealed an obvious lack of normality and. furthermore, their means tended to reach zero in a few days ( 3 or 4 ); thus, for this analysis, the appropriate model was a nonparametric one. A Kruskal-Wallis test was performed comparing days of testing within sexes and housing conditions, and housing conditions within sexes and days.

After this first statistical analysis, a second set of ANOVAs was performed using the same main factors but including only the data obtained from Days 3-10. The objective of this new analysis was to assess the relative importance of some effects which were clearly distinguishable in the figures, but which did not reach significance in the previous statistical tests.

When a significant interaction was found in any ANOVA, a Scheffe multiple comparisons test was performed to analyze that interaction.

The significance level was fixed at .05 in order to keep Type II error probability as low as possible.

\section{RESULTS}

\section{Acquisition Phase}

The analysis of the number of AVO responses during the whole experimental period revealed significant effects
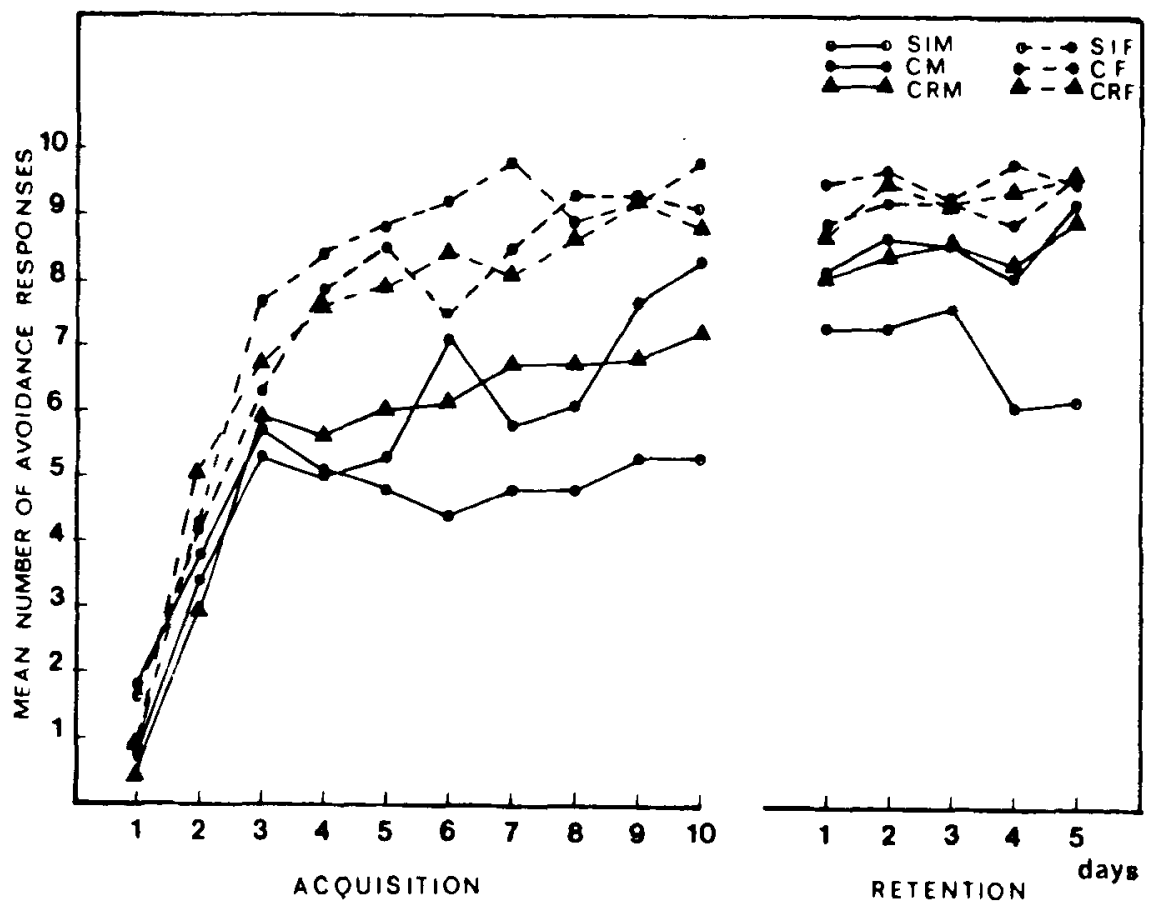

Figure 1. Changes in the avoidance response of each experimental group during the two phases of the test (acquisition and retention). SIM = socially isolated males, $\mathbf{C M}=$ control males, $\mathbf{C R M}$ $=$ crowded males, SIF $=$ socially isolated females, $\mathrm{CF}=$ control females, $\mathrm{CRF}=$ crowded females. 
of the three main factors [housing condition, $F(2,650)=$ $6.11, p<.01 ; \operatorname{sex}, F(1,650)=161.98, p<.001$; and trials, $F(9,650)=65.16, p<.001]$. A highly significant interaction between sex and trials was also found $[F(9,650)=16.08, p<.001]$; however, the remaining interactions were not significant.

Differences between treatments are attributable to the poorer performance of the isolated animals; however, no differences could be detected between the control and the crowded groups of either sex. Female rats in the three different rearing conditions showed a significantly greater number of AVO responses than did their male counterparts. The sex $x$ trials interaction was a consequence of the different patterns of responses of males and females to the learning process, which can be observed in Figure 1; females showed an increasingly higher mean during the acquisition phase.

The analysis of ESC responses in the same period (Days 1-10) led us to the same conclusions as the analysis of AVO responses, except for the lack of significance of housing conditions. The main effects of sex and trials were highly significant, as was the sex $\times$ trials interaction. The other interactions were not significant. These results can be described as above (AVO responses), just by changing the sign of the inequalities; that is, females showed lower means, which decreased more quickly than those for their male counterparts (Figure 2).

Errors were first analyzed within sexes and housing conditions. All groups revealed highly significant differences across days, except that errors made by the isolated males did not decline significantly over trials. A further analysis within sexes and days revealed that isolated females made more errors than the other two female groups on Day 2 of testing $\left[\chi^{2}(2)=7.30, p<.05\right]$, whereas isolated males made significantly fewer errors than the two other male groups on Day 1 of the test $\left[\chi^{2}(2)=\right.$ $11.72, p<.01$ ] (Figure 3 ). On later days, the only significant difference appeared on Day 9 among male rats, isolates showing a higher $\mathrm{E}$ score than the two other groups $\left[\chi^{2}(2)=6.53, p<.05\right]$.

A further ANOVA was performed for Days 3-10, in order to determine whether the apparent interaction between sex and housing condition for AVO responses (Figure 1), which was not significant over all days, would reach significance when very early acquisition was eliminated from the data. The analysis of AVO responses during this period confirmed the significant effects of the three main factors [housing condition, $F(2,520)=9.15$, $p<.001$; sex, $F(1,520)=164.81, p<.001$; and trials, $F(7,520)=26.15, p<.001]$. The interaction betwen sex and trials did not reach significance in this analysis $[F(7,520)=1.05, p=.4]$ as it did in the others. However, the housing condition $x$ sex interaction was highly significant $[F(2,520)=4.09, p<.001]$. This latter result was a function of housing conditions affecting male, but not female, rats. The subsequent multiple comparisons test indicated a lessened AVO response in the SIM group when compared with each of the other male groups.

The analysis of ESC responses yielded similar results, the main difference being the lack of significance of housing conditions. As with AVO responses, the sex $\times$ hous-

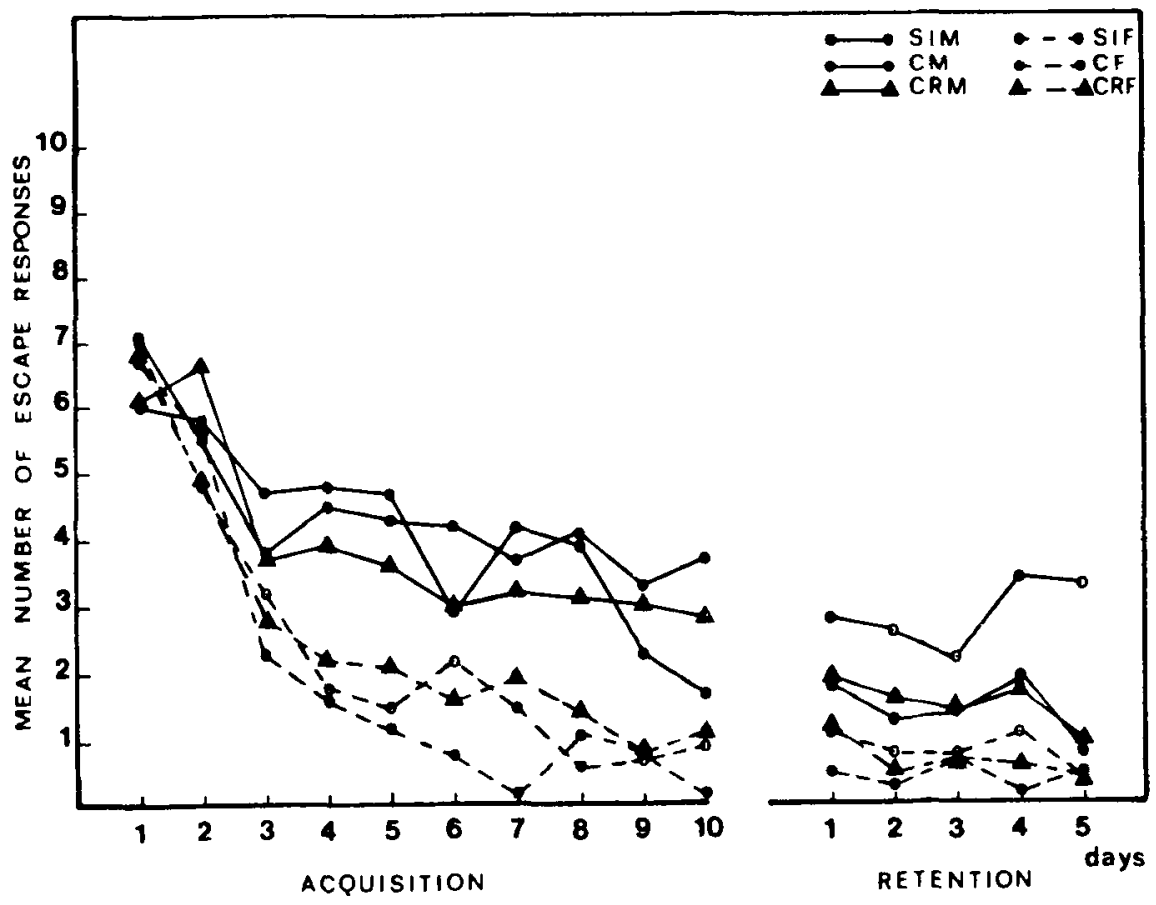

Figure 2. Changes in the escape response of each experimental group during the two phases of the test (acquisition and retention). SIM = socially isolated males, CM = control males, CRM = crowded males, SIF = socially isolated females, CF $=$ control females, CRF $=$ crowded females. 


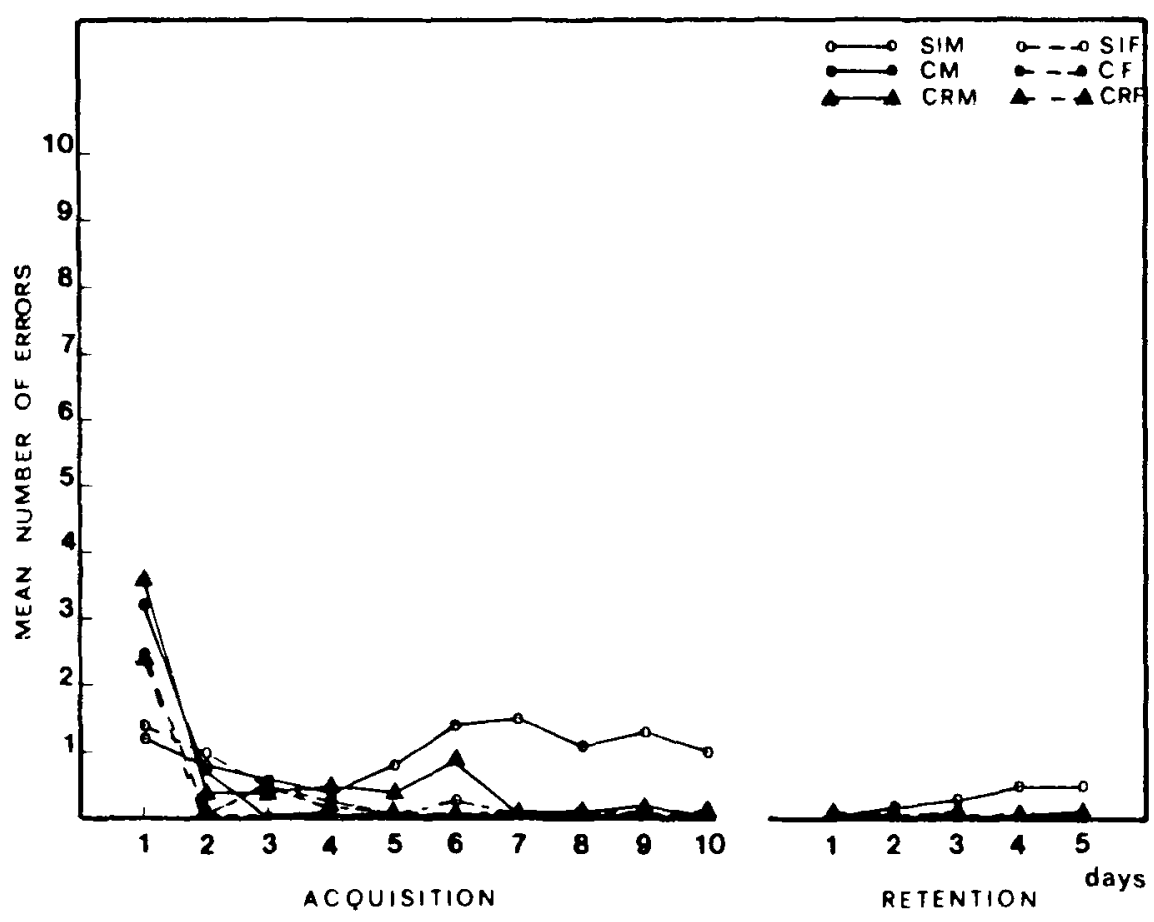

Figure 3. Changes in the error rate of each experimental group during the two phases of the test (acquisition and retention). SIM = socially isolated males, $\mathrm{CM}=$ control males, $\mathrm{CRM}=$ crowded males, SIF = socially isolated females, $\mathrm{CF}=$ control females, CRF $=$ crowded females.

ing condition interaction was significant; nevertheless, no significant differences were observed between rats reared in different social conditions within each gender.

The effects of the three main factors on Def scores were highly significant (Figure 4) during the whole acquisition phase [housing condition, $F(2,650)=76.33, p<.001$; sex, $F(1,650)=160.19, p<.001$; and trials, $F(9,650)$ $=7.81, p<.001]$. Significant interactions were found between housing conditions and sex, as well as between sex and trials $[F(2,650)=8.02, p<.001$, and $F(9,650)=$ $6.78, p<.001$, respectively]. The two other interactions were not significant. Post hoc comparisons among the male groups indicated a higher Def score in the crowded rats than in both the control and the isolated groups, whereas no significant difference was found between the isolated and control males. On the contrary, among females, the isolated rats showed a lower Def score than did the two other groups, whereas control and crowded females did not differ. It should be noted that the CRM group appeared to be considerably more emotional than the SIM group on this measure; however, paradoxically, the SIM group showed a lower AVO response, as mentioned above.

Within each social condition, there was a higher Def score in the males than in the females.

\section{Retention Phase}

In the analysis of AVO responses during the retention phase, only two factors-housing conditions and sexremained significant $[F(2,325)=15.27, p<.001$, and
$F(1,325)=64.74, p<.001$, respectively], and no effect of trials was apparent, as expected. In contrast to the acquisition phase, at this stage of the test, learning had already been achieved and thus both responses were relatively stable. Female rats made more AVO responses than did males. Once again, the housing condition $\times$ sex interaction was significant $[F(2,325)=6.73, p<.01]$, housing conditions affecting male, but not female, rats. The Scheffé test revealed significantly fewer AVO responses in the SIM group than in CM and CRM groups; there were no differences between these latter two groups.

With regard to the ESC response, a significant effect of housing conditions appeared during this period $[F(2,325)=12.92, p<.001]$. In relation to this result, the analysis of errors did not reveal any significant difference, since the individual $\mathrm{E}$ scores were always equal to zero. There was a significant effect of sex $[F(1,325)=$ $65.05, p<.001]$ and a significant rearing condition $x$ sex interaction $[F(2,325)=4.58, p<.05]$. The remaining interactions were not significant. With this variable as well, the housing condition $\times$ sex interaction was a function of housing condition affecting males but not females. The multiple comparisons test revealed more ESC responses in the SIM group than in the CM and CRM groups.

In relation to the Def score, as in the previous period of the experiment, the three main factors involved were highly significant [housing condition, $F(2,325)=49.13$, $p<.001$; sex, $F(1,325)=111.82, p<.001$; and trials, $F(4,325)=7.86, p<.001]$. The housing condition $\times$ sex 


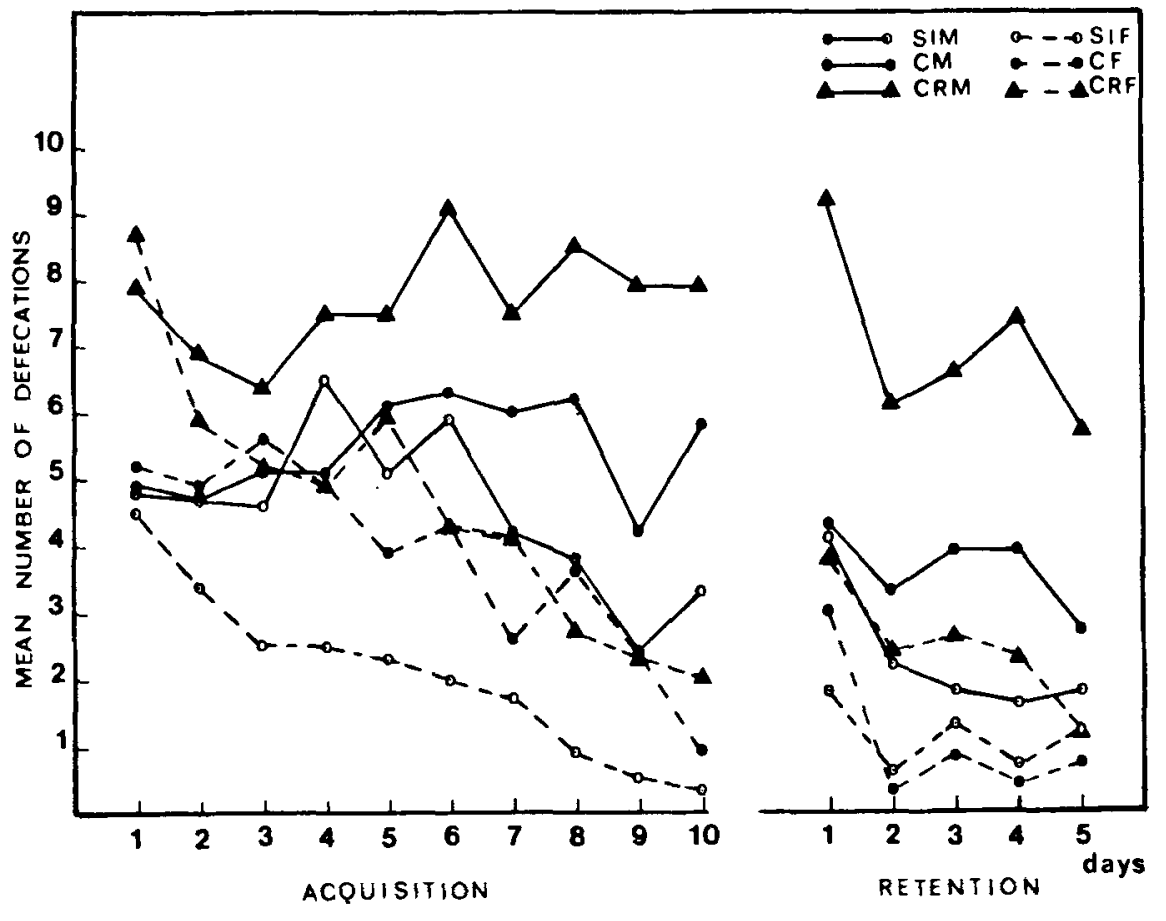

Figure 4. Changes in the defecation score of each experimental group during the two phases of the test (acquisition and retention). SIM = socially isolated males, $\mathbf{C M}=$ control males, $\mathbf{C R M}=$ crowded males, SIF = socially isolated females, CF = control females, CRF = crowded females.

interaction reached a higher significance level in the second phase $[F(2,325)=15.98, p<.001]$, although the other interactions remained nonsignificant. Among males, the crowded animals again showed the highest mean value, which was significantly different from the two other groups; the Def score of the isolates was significantly lower than that of the control group in this period. Crowded females showed higher Def scores than did isolated and control rats of the same gender, but the latter two groups did not differ.

\section{DISCUSSION}

The present results suggest a greater vulnerability of male rats to the social treatments. Social isolation impaired avoidance performance among males, whereas no significant influence of the social treatments was observed in the performance of the females throughout the test.

In agreement with previous reports (e.g., Gray \& Lalljee, 1974), the present results also indicate that female rats are superior to males in this learning paradigm. Furthermore, the social treatments did not eliminate sex differences. During the acquisition phase, the females showed a rapid decrease in their escape responses as early as Day 2 of the test, concomitant with a continuous increase in avoidance responses. Male groups, in contrast, showed a slower reduction in their escape responses, with a delayed acquisition of the avoidance response. During the retention period, females still displayed significantly more avoidance responses and fewer escapes than did their male counterparts. These results suggest the important role that gonadal hormones may play in this learning paradigm (Manshio \& Gershbein, 1975). A relationship between the emotional level of the animals and such sex differences has been claimed (Gray, 1979; Gray \& Lalljee, 1974), although other authors do not uphold this idea (Archer, 1971, 1973). The lower defecation score shown by the female rats might support the first hypothesis. In this respect, our results agree with Wilcock and Fulker's (1973) model based on the distinction between two different processes in this type of conditioning, the first under the influence of the conditioned emotional response, which may lead to the immobility ("freezing") of the animal, and the second characterized by an improvement in avoidance performance.

It is worth emphasizing that the influence of gonadal hormones responsible for the sex differences in performance on this test (i.e., a superior avoidance performance associated with a lower defecation score in females) appears to be stronger than that of the housing conditions, which did not eliminate those sex differences. However, when only male groups are considered, the apparently lower emotionality (in terms of defecation score) is dissociated from superior avoidance learning. A number of points should be considered that may account for this apparent paradox, that is, a lower defecation score appearing associated with impaired avoidance performance. Thus, a differential effect of both social treatments on learning performance, as well as on the defecation score of the males, has been found. In fact, social isolation induced an impairment of the avoidance performance of the animals together with a decrease in their defecation scores 
(retention phase). In contrast, the crowding treatment did not produce significant effects on the learning ability of the male rats, although it increased their defecation score. Thus, as pointed out in the previous section, the crowded males showed a higher defecation score and yet a superior avoidance performance, relative to the isolates. These results suggest that the two extreme housing conditions used in this work involve two different kinds of stress, which may affect different neuroendocrine systems in the treated animals (see Viveros et al., 1988).

Social isolation appeared to be the more effective treatment in disturbing the learning mechanisms of the male rats in this test. The isolated rats showed the fewest avoidance responses and the most escape responses and errors combined. These results are in accordance with previous findings of impaired learning ability in isolates, as demonstrated in other tests (Dalrymple-Alford \& Benton, 1984; Joseph \& Gallager, 1980) as well as in this kind of conditioning (Will \& Kelche, 1979). There arè, however, some discrepancies in the literature concerning the present learning paradigm. A number of methodological features might account for some of these differences. Lovely et al. (1972) reported a facilitating effect of individual housing on acquisition and extinction of the avoidance response in male rats. The discrepancies in the present results might be due to the shorter isolation treatment used here, which was, furthermore, imposed on adult rats. Moreover, the isolates in Lovely et al.'s work were compared with animals that had been housed in groups of 15; we feel that this exceeds the number commonly considered as a standard for a control group (4-6 animals per cage in the present work).

It is generally accepted that rearing rats in restricted environments causes a certain perseverating tendency to repetitious patterns of behavior (Joseph \& Gallager, 1980 ), which obviously results in a deficient learning ability. This might account for the tendency of isolated males to maintain their escape response at the expense of a delay in acquisition of an avoidance response. Thus, social isolation appears to damage the ability of rats to change the timing of their response to a more adaptive one which would prevent the noxious stimulus. Although this kind of deficit appears to be complex (Morgan \& Einon, 1975), it has been suggested that a number of responses that are specifically under inhibitory control (e.g., the extinction of a previously rewarded response) may be impaired by a postweaning isolation treatment (Dalrymple-Alford \& Benton, 1984).

We have recently reported decreased adrenocortical reactivity in isolated males (Viveros et al., 1988), indicating an impaired flexibility of their adrenal response (see also Plaut \& Grota, 1971). This modification of adrenal function might underlie the lack of behavioral flexibility necessary to change the timing of their response, as shown by the male isolates in the present work. Moreover, the fact that the above-mentioned modifications were still present in the retention phase seems to support the existence of a perseverating, maladaptive deficit in socially isolated males. Possible damage in certain disinhibitory brain areas (Morgan, Einon, \& Morris, 1977) and/or insufficient neuroanatomical development produced by the deprivation of social experience over a relatively long time period (Rosenzweig et al., 1978; Welch et al., 1974) might underlie the behavioral alterations observed in these animals.

Further research is needed to clarify the interaction between the differential effects of social isolation and crowding on treated animals and the neuroendocrine systems involved, particularly those affecting sex differences.

\section{REFERENCES}

ARCHER, J. (1971). Sex differences in emotional behavior: A reply to Gray and Buffery. Acta Psychologica, 35, 415-429.

ARCHER, J. (1973). Tests for emotionality in rats and mice: A review Animal Behaviour, 21, 205-235.

Armario, A., Castellanos, J. M., \& Balasch, J. (1984). Effect of crowding on emotional reactivity in male rats. Neuroendocrinology, 39, 330-333.

Dalrymple-Alford, J. C., Benton, D. (1984). Behavioral inhibition and the age at social isolation in rats. Quarterly Journal of Experimental Psychology, 36B, 27-38.

EClANCHER, F., KARLY, P. (1980). Effects of infant and adult amygdaloid lesions upon acquisition of two-way active avoidance by the adult rat: Influence of rearing conditions. Physiology \& Behavior, 24, 887-893.

GrAY, J. A. (1979). Emotionality in male and female rodents: A reply to Archer. British Journal of Psychology, 70, 425-440.

Gray, J. A., LALLJEe, B. (1974). Sex differences in emotional behaviour in the rat: Correlation between open-field defecation and active avoidance. Animal Behaviour, 22, 856-861.

JosePH, R. (1979). Effects of rearing and sex on maze learning and competitive exploration in rats. Joumal of Psychology, 101, 37-43.

JosePH, R., \& GAllaGer, R. E. (1980). Gender and early environmental influences on activity, overresponsiveness and exploration. Developmental Psychobiology, 13, 527-544.

Juraska, J. M., Henderson, C., Müller, J. (1984). Differential rearing experience, gender, and radial maze performance. Developmental Psychobiology, 17, 209-215.

Lovely, R. H., Pagano, R. R., * Paolino, R. M. (1972). Shuttlebox-avoidance performance and basal corticosterone levels as a function of duration of individual housing in rats. Joumal of Comparative \& Physiological Psychology, 81, 331-335.

Manshio, D. T., \& Gershbein, L. L. (1975). Avoidance and poke behavior in rats after gonadectomy and hormonal treatment. Research Communications in Chemical Pathology \& Pharmacology, 12, 473-479.

Morgan, M., Einon, D. (1975). Incentive motivation and behavioral inhibition in socially-isolated rats. Physiology \& Behavior, 15, 405-409.

Morgan, M. J., Einon, D., \& Morris, R. G. M. (1977). Inhibition and isolation rearing in the rat: Extinction and satiation. Physiology \& Behavior, 18, 1-5.

Plaut, S. M., Grota, L. J. (1971). Effects of differential housing on adrenocortical reactivity. Neuroendocrinology, 7, 348-360.

Rosenzweig, M. R., Bennett, E. L., Hebert, M., Morimato, H. (1978). Social grouping cannot account for cerebral effects of enriched environments. Brain Research, 153, 563-576.

Thiebot, M. H., Soubrue, P., Chermat, R., Simon, P., \& Boissier, J. R. (1977). Caractéristiques comportementales et psychopharmacologiques de rats élevés en haute densité de population. Psychopharmacology, 54, 283-288.

Viveros, M. P., Hernández, R., Martínez, I., \& González, P. (1988). Effects of social isolation and crowding upon adrenocortical reactivity and behavior in the rat. Revista Espanola de Fisiologia, 44, 315-322. 
Welch, B. L., Brown, D. G., Welch, A. S., LiN, D. C. (1974). Isolation, restrictive confinement or crowding of rats for one year: 1. Weight, nucleic acids and protein of brain regions. Brain Research, 75, 71-84.

WILcock. J., \& Fulker. D. W. (1973). Avoidance learning in rats: Genetic evidence for two distinct behavioral processes in the shuttlebox. Journal of Comparative \& Physiological Psychology, 82, 247-253.
WiLl, B. E., Kelche, C. R. (1979). Effects of different postoperative environments on the avoidance behavior of rats with hippocampal lesions: Recovery or improvement of function? Behavioral \& Neural Biology, 27, 96-106.

(Manuscript received January 19, 1989; revision accepted for publication February 19, 1989.) 